\title{
Two-Fluid Atmosphere for Relativistic Stars
}

\author{
E.N. Glass* and J.P. Krisch \\ Department of Physics, University of Michigan, Ann Arbor, Michgan 48109
}

(December 21, 1998)

\begin{abstract}
We have extended the Vaidya radiating metric to include both a radiation fluid and a string fluid. This paper expands our brief introduction to extensions of the Schwarzschild vacuum which appeared in 1998 Phys. Rev. D 57 R5945. Assuming diffusive transport for the string fluid, we find new analytic solutions of Einstein's field equations.
\end{abstract}

PACS numbers: 04.20.Jb, 04.40.Dg, 97.60.-s

*Permanent address: Physics Department, University of Windsor, Ontario N9B 3P4, Canada 


\section{INTRODUCTION}

Strings have become a very important ingredient in many physical theories. They may have been present in the early universe and played a role in the seeding of density inhomogeneities [1]. The idea of strings is fundamental to superstring theories [2]. String fluids have been suggested as dark matter candidates. The lensing properties of cosmic strings and string systems have been discussed [3] and the apparent relationship between counting string states and the entropy of the Schwarzschild horizon [4], [5], [6] suggests an association of strings with black holes. Recently Glass and Krisch [7] have pointed out that allowing the Schwarzschild mass parameter to be a function of radial position creates an atmosphere with a string fluid stress-energy around a static, spherically symmetric, object. If the mass is also a function of retarded time a Vaidya radiation fluid is present in addition to the string fluid. Since the metric has one arbitrary function, $m(u, r)$, invariantly defined by the sectional curvature of the $u, r$ two-surfaces, a given mass distribution determines the stress-energy.

The string fluid stress-energy is a macroscopic, statistically averaged, description of a microscopic distribution of Planck length string bits. The string fluid lies on a two-dimensional timelike world-sheet $(u, r)$ and its stress-energy is parametrized by radial derivatives of the mass function. The radial stress, $p_{r}<0$, is the expected string tension. Since the string fluid is constrained to stay in the $(u, r)$ plane, the Planck scale string bits have only radial motions. Interactions of the string bits along the radial directions are modeled by the macroscopic string tension $p_{r}$ and create, on average, a long string stretching radially away from the core or horizon of the mass distribution. The Glass-Krisch atmosphere allows transverse stresses. These stresses, if present, can be modeled by the presence of a pressureless dust fluid. The transverse pressures arise from the different velocities of the dust and string fluids. The most general atmosphere has three components: a Vaidya radiation fluid, a string fluid with radial tension, and a dust fluid. This can be interpreted as a two-fluid atmosphere with 
a transverse pressure. We also discuss other, less general, possibilities.

This atmosphere can model a variety of physical situations at different distance scales. It can describe the atmosphere around a black hole with a distance scale of multiples of Schwarzschild radii. It could also describe a globular cluster with a dark matter component and a distance scale of the order of parsecs.

The contracted Bianchi identities are satisfied for arbitrary $m(u, r)$ and so the mode of mass propagation is a modeling choice from the many possible propagation equations of classical and quantum physics. Each choice allows the generation of new analytic solutions to the field equations. In this work we develop new solutions by considering diffusive transport of the string fluid elements. With this choice we find that the string fluid diffuses inward as the Vaidya photons carry energy outward. The overall effect is to slow down the time scale of the Vaidya energy loss.

In the next section we present our extension of Vaidya's metric [8] as a further extension of the Schwarzschild vacuum solution. The arbitrary mass function, which appears in the metric, is identified as the sectional curvature of nested two-spheres. The string fluid and an associated static two-fluid model are described in the third and fourth sections. We are able to provide a two-fluid kinematic interpretation of the transverse stresses in this model. In the fifth part of the paper diffusive transport is developed and analytic solutions for the energy density are found. In the sixth section analytic mass solutions are presented and interpreted. The last two sections contain the analysis of the horizon structure of some of our solutions and a discussion of the general results.

In this work Greek indices range over $(0,1,2,3)=(u, r, \vartheta, \varphi)$. Our sign conventions are $2 A_{\nu ;[\alpha \beta]}=A_{\mu} R_{\nu \alpha \beta}^{\mu}$, and $R_{\mu \nu}=R_{\mu \nu \alpha}^{\alpha}$. Overdots abbreviate $\partial / \partial u$, and primes abbreviate $\partial / \partial r$. Overhead carets denote unit vectors. We use units where $G=c=1$. Einstein's field equations are $G_{\mu \nu}=-8 \pi T_{\mu \nu}$, and the metric signature is $(+,-,-,-)$. 


\section{EXTENDING THE SCHWARZSCHILD VACUUM}

The spacetime metric covering the region exterior to a spherical star is given by

$$
d s^{2}=A d u^{2}+2 d u d r-r^{2}\left(d \vartheta^{2}+\sin ^{2} \vartheta d \varphi^{2}\right)
$$

where $A=1-2 m(u, r) / r$. Initially $m(u, r)=m_{0}$ provides the vacuum Schwarzschild solution in the region $r>2 m_{0}$. At later times that region admits a two-fluid description of radial strings and outward flowing short-wavelength photons (sometimes called a "null fluid"). Metric (11) is spherically symmetric and given in retarded time coordinate $u$. With the use of a Newman-Penrose null tetrad the Einstein tensor is computed from (1) and given by

$$
\begin{aligned}
G_{\mu \nu}= & -2 \Phi_{11}\left(l_{\mu} n_{\nu}+n_{\mu} l_{\nu}+m_{\mu} \bar{m}_{\nu}+\bar{m}_{\mu} m_{\nu}\right) \\
& -2 \Phi_{22} l_{\mu} l_{\nu}-6 \Lambda g_{\mu \nu} .
\end{aligned}
$$

Here the null tetrad components of the Ricci tensor are

$$
\begin{aligned}
\Phi_{11} & =\left(2 m^{\prime}-r m^{\prime \prime}\right) /\left(4 r^{2}\right), \\
\Phi_{22} & =-\dot{m} / r^{2}, \\
\Lambda & =R / 24=\left(r m^{\prime \prime}+2 m^{\prime}\right) /\left(12 r^{2}\right) .
\end{aligned}
$$

The only non-zero component of the Weyl tensor is

$$
\Psi_{2}=-m / r^{3}+\left(4 m^{\prime}-r m^{\prime \prime}\right) /\left(6 r^{2}\right)
$$

The metric is Petrov type $\mathbf{D}$ with $l_{\mu}$ and $n_{\mu}$ principal null geodesic vectors

$$
\begin{aligned}
l_{\mu} d x^{\mu} & =d u \\
n_{\mu} d x^{\mu} & =(A / 2) d u+d r \\
m_{\mu} d x^{\mu} & =-(r / \sqrt{ } 2)(d \vartheta+i \sin \vartheta d \varphi),
\end{aligned}
$$

where 


$$
\begin{aligned}
l_{\mu ; \nu} & =\left(A^{\prime} / 2\right) l_{\mu} l_{\nu}-(1 / r)\left(m_{\mu} \bar{m}_{\nu}+\bar{m}_{\mu} m_{\nu}\right), \\
n_{\mu ; \nu} & =-\left(A^{\prime} / 2\right) n_{\mu} l_{\nu}+(A / 2 r)\left(m_{\mu} \bar{m}_{\nu}+\bar{m}_{\mu} m_{\nu}\right), \\
m_{\mu ; \nu} & =(A / 2 r) l_{\mu} m_{\nu}-(1 / r) n_{\mu} m_{\nu}+(\cot \vartheta / \sqrt{2} r)\left(m_{\mu} m_{\nu}-m_{\mu} \bar{m}_{\nu}\right) .
\end{aligned}
$$

In order to clearly see the two-fluid description we introduce a timelike unit velocity vector $\hat{v}^{\mu}$ and three unit spacelike vectors $\hat{r}^{\mu}, \hat{\vartheta}^{\mu}, \hat{\varphi}^{\mu}$ such that

$$
g_{\mu \nu}=\hat{v}_{\mu} \hat{v}_{\nu}-\hat{r}_{\mu} \hat{r}_{\nu}-\hat{\vartheta}_{\mu} \hat{\vartheta}_{\nu}-\hat{\varphi}_{\mu} \hat{\varphi}_{\nu}
$$

The unit vectors are defined by

$$
\begin{array}{ll}
\hat{v}_{\mu} d x^{\mu}=A^{1 / 2} d u+A^{-1 / 2} d r, \quad \hat{v}^{\mu} \partial_{\mu}=A^{-1 / 2} \partial_{u}, \\
\hat{r}_{\mu} d x^{\mu}=A^{-1 / 2} d r, & \hat{r}^{\mu} \partial_{\mu}=A^{-1 / 2} \partial_{u}-A^{1 / 2} \partial_{r}, \\
\hat{\vartheta}_{\mu} d x^{\mu}=r d \vartheta, & \hat{\vartheta}^{\mu} \partial_{\mu}=-r^{-1} \partial_{\vartheta}, \\
\hat{\varphi}_{\mu} d x^{\mu}=r \sin \vartheta d \varphi, & \hat{\varphi}^{\mu} \partial_{\mu}=-(r \sin \vartheta)^{-1} \partial_{\varphi} .
\end{array}
$$

$\hat{v}^{\mu}$ is hypersurface-orthogonal, i.e. $\hat{v}_{[\mu ; \nu} \hat{v}_{\alpha]}=0$, with $h_{\mu \nu}$ the first fundamental form of the hypersurface. Since $\hat{v}_{\mu} d x^{\mu}=f(u, r) d t$, the components of $h_{\mu \nu}$ show explicitly that $\hat{v}^{\mu}$ lies along $t=$ const time lines:

$$
\begin{aligned}
h_{\mu \nu} d x^{\mu} d x^{\nu} & =\left(g_{\mu \nu}-\hat{v}_{\mu} \hat{v}_{\nu}\right) d x^{\mu} d x^{\nu} \\
& =-A^{-1} d r^{2}-r^{2}\left(d \vartheta^{2}+\sin ^{2} \vartheta d \varphi^{2}\right) .
\end{aligned}
$$

The kinematics of the $\hat{v}^{\mu}$ flow are described by

$$
\hat{v}_{; \nu}^{\mu}=a^{\mu} \hat{v}_{\nu}+\sigma^{\mu}{ }_{\nu}-(\Theta / 3)\left(\hat{r}^{\mu} \hat{r}_{\nu}+\hat{\vartheta}^{\mu} \hat{\vartheta}_{\nu}+\hat{\varphi}^{\mu} \hat{\varphi}_{\nu}\right),
$$

where

$$
\begin{aligned}
a^{\mu} & =\left[\dot{m} / r+A \partial_{r}(m / r)\right] A^{-3 / 2} \hat{r}^{\mu}, \\
\sigma^{\mu}{ }_{\nu} & =(\Theta / 3)\left(-2 \hat{r}^{\mu} \hat{r}_{\nu}+\hat{\vartheta}^{\mu} \hat{\vartheta}_{\nu}+\hat{\varphi}^{\mu} \hat{\varphi}_{\nu}\right), \\
\Theta & =(\dot{m} / r) A^{-3 / 2} .
\end{aligned}
$$


The Einstein tensor can now be written as a two-fluid system:

$$
\begin{aligned}
G_{\mu \nu}= & \left(2 \dot{m} / r^{2}\right) l_{\mu} l_{\nu}-\left(2 m^{\prime} / r^{2}\right)\left(\hat{v}_{\mu} \hat{v}_{\nu}-\hat{r}_{\mu} \hat{r}_{\nu}\right) \\
& +\left(m^{\prime \prime} / r\right)\left(\hat{\vartheta}_{\mu} \hat{\vartheta}_{\nu}+\hat{\varphi}_{\mu} \hat{\varphi}_{\nu}\right) .
\end{aligned}
$$

Spherical symmetry allows the function $m(u, r)$ to be identified as the mass within twosurfaces of constant $u$ and $r$, and invariantly defined from the sectional curvature [28] of those surfaces:

$$
-2 m / r^{3}=R_{\alpha \beta \mu \nu} \hat{\vartheta}^{\alpha} \hat{\varphi}^{\beta} \hat{\vartheta}^{\mu} \hat{\varphi}^{\nu}
$$

\section{STRING FLUID}

The string bivector is defined by

$$
\Sigma^{\mu \nu}=\epsilon^{B C} \frac{\partial x^{\mu}}{\partial x^{B}} \frac{\partial x^{\nu}}{\partial x^{C}}, \quad(B, C)=(0,1) \text { or }(2,3)
$$

Spherical symmetry demands that the averaged string bivector will have a world-sheet in either the $(u, r)$ or $(\vartheta, \varphi)$ plane. The condition that the world-sheets are timelike, i.e. $\gamma:=\frac{1}{2} \Sigma^{\mu \nu} \Sigma_{\mu \nu}<0$, implies that only the $\Sigma_{u r}$ component is non-zero. It is useful to write $\Sigma^{\mu \nu}$ in terms of unit vectors

$$
\Sigma^{\mu \nu}=\hat{r}^{\mu} \hat{v}^{\nu}-\hat{v}^{\mu} \hat{r}^{\nu}
$$

It is now clear that $\Sigma^{\mu \alpha} \Sigma_{\alpha}^{\nu}=\hat{v}^{\mu} \hat{v}^{\nu}-\hat{r}^{\mu} \hat{r}^{\nu}$. We follow Letelier [9], [10] and write a string energy-momentum tensor by analogy with one for a perfect fluid

$$
T_{\mu \nu}^{f l u i d}=\rho u_{\mu} u_{\nu}-p h_{\mu \nu}
$$

where $h^{\mu}{ }_{\nu}=\delta^{\mu}{ }_{\nu}-u^{\mu} u_{\nu}, h^{\mu}{ }_{\nu} u^{\nu}=0$. The string energy-momentum is given by

$$
T_{\mu \nu}^{\text {string }}=\rho(-\gamma)^{1 / 2} \hat{\Sigma}_{\mu}^{\alpha} \hat{\Sigma}_{\alpha \nu}-p_{\perp} H_{\mu \nu}
$$


where $H_{\nu}^{\mu}=\delta_{\nu}^{\mu}-\hat{\Sigma}^{\mu \alpha} \hat{\Sigma}_{\alpha \nu}, H_{\nu}^{\mu} \hat{\Sigma}^{\nu \beta}=0$. Although here $\gamma=-1$, we have kept $\gamma$ explicit in (14) and written $\hat{\Sigma}^{\mu \nu}:=(-\gamma)^{-1 / 2} \Sigma^{\mu \nu}$ to show how $\hat{\Sigma}^{\mu \nu}$ is made invariant to reparameterizations of the world-sheets [9].

Einstein's field equations allow the matter portion of $G_{\mu \nu}$ in Eq.(11) to be identified as a string fluid:

$$
\begin{aligned}
T_{\mu \nu} & =T_{\mu \nu}^{r a d}+T_{\mu \nu}^{s t r i n g} \\
& =\psi l_{\mu} l_{\nu}+\rho \hat{v}_{\mu} \hat{v}_{\nu}+p_{r} \hat{r}_{\mu} \hat{r}_{\nu}+p_{\perp}\left(\hat{\vartheta}_{\mu} \hat{\vartheta}_{\nu}+\hat{\varphi}_{\mu} \hat{\varphi}_{\nu}\right)
\end{aligned}
$$

Thus

$$
\begin{aligned}
4 \pi \psi & =-\dot{m} / r^{2}, \\
4 \pi \rho & =-4 \pi p_{r}=m^{\prime} / r^{2}, \\
8 \pi p_{\perp} & =-m^{\prime \prime} / r .
\end{aligned}
$$

Since the contracted Bianchi identities are satisfied for arbitrary $m(u, r)$, it follows that the

equations of motion $T_{; \nu}^{\mu \nu}=0$ are identically satisfied for the components of $T_{\mu \nu}$ given in Eq.(15).

The components of the contracted Bianchi identities which do not vanish because of spherical symmetry but rather because of the explicit form $A=1-2 m(u, r) / r$ are

$$
l_{\mu} G_{; \nu}^{\mu \nu}=-\nabla_{\nu}\left[\left(2 \Phi_{11}+R / 4\right) l^{\nu}\right]-G^{\mu \nu} l_{\mu ; \nu}
$$

and

$$
n_{\mu} G_{; \nu}^{\mu \nu}=-\nabla_{\nu}\left[\left(2 \Phi_{11}+R / 4\right) n^{\nu}+\Phi_{22} l^{\nu}\right]-G^{\mu \nu} n_{\mu ; \nu} .
$$

\section{STATIC FLUID MODELS}

Static models are useful in developing insights about time dependent fluids. We consider two static models that are equivalent to the general stress-energy tensor described in Eq.(15) when it is time-independent. The first is a static isotropic string fluid, and the second provides a two-fluid interpretation of (15) and an interpretation of the transverse stress. 


\section{A. Isotropic string fluid}

Consider the stress-energy tensor in Eq.(15) with static mass function $m(r)$ and with $p_{r}=p_{\perp}:$

$$
T_{\mu \nu}^{i s o}=-p_{r}\left(\hat{v}_{\mu} \hat{v}_{\nu}-\hat{r}_{\mu} \hat{r}_{\nu}-\hat{\vartheta}_{\mu} \hat{\vartheta}_{\nu}-\hat{\varphi}_{\mu} \hat{\varphi}_{\nu}\right)
$$

This is clearly an isotropic cloud of strings with equation of state $\rho+p_{r}=0$. The mass is determined by the constraint $p_{r}=p_{\perp}$ or

$$
\frac{m^{\prime \prime}}{2 r}=\frac{m^{\prime}}{r^{2}}
$$

The solution of Eq.(18), in the more general time-dependent case with null radiation fluid, is

$$
m(u, r)=r^{3} c_{1}(u)+c_{2}(u)
$$

For the static case we have $c_{1}$ and $c_{2}$ constant. As can be seen from energy-momentum Eq.(17) the isotropic string cloud is in an Einstein spacetime.

\section{B. Static two-fluid model}

One can use two different four-velocities, $\hat{u}_{\mu}$ and $\hat{w}_{\mu}$, to write a two-fluid model:

$$
T_{\mu \nu}^{2 \text { fluid }}=\rho_{2} \hat{w}_{\mu} \hat{w}_{\nu}+\rho_{1} \hat{u}_{\mu} \hat{u}_{\nu}+p_{r} \hat{r}_{\mu} \hat{r}_{\nu}
$$

The fluid with $\rho_{2}$ is dust and the other is a fluid with a radial stress. $(\dot{m}=0$ and there is no Vaidya radiation fluid.) Letelier [15] has described a procedure for casting two-fluid stress-energies into the form of an anisotropic fluid. His method is not adapted for the string fluid equation of state. As a variation of his method, we transform the fluid velocities to create two un-normalized vectors: $V_{\mu}$ timelike and $Y_{\mu}$ spacelike.

$$
\begin{aligned}
& \sqrt{\rho_{1}} V_{\mu}=\sqrt{\rho_{1}} \cos \alpha \hat{u}_{\mu}+\sqrt{\rho_{2}} \sin \alpha \hat{w}_{\mu} \\
& \sqrt{\rho_{2}} Y_{\mu}=-\sqrt{\rho_{1}} \sin \alpha \hat{u}_{\mu}+\sqrt{\rho_{2}} \cos \alpha \hat{w}_{\mu} .
\end{aligned}
$$


This transformation obeys

$$
\rho_{1} \hat{u}_{\mu} \hat{u}_{\nu}+\rho_{2} \hat{w}_{\mu} \hat{w}_{\nu}=\rho_{1} V_{\mu} V_{\nu}+\rho_{2} Y_{\mu} Y_{\nu}
$$

Since $Y_{\mu}$ is spacelike, Eq.(22) is valid only for $\hat{u}_{\mu} \neq \hat{w}_{\mu}$ and $\alpha \neq n(\pi / 2)$. The parameter $\alpha$ is defined by $V_{\mu} Y^{\mu}=0$ :

$$
\cot 2 \alpha=\frac{\rho_{1}-\rho_{2}}{2 \sqrt{\rho_{1} \rho_{2}} \hat{u}_{\mu} \hat{w}^{\mu}}
$$

The stress-energy $T_{\mu \nu}^{2 \text { fluid }}$ can be written as

$$
T_{\mu \nu}^{\text {transf }}=\rho \hat{V}_{\mu} \hat{V}_{\nu}+p_{r} \hat{r}_{\mu} \hat{r}_{\nu}+p_{\perp} \hat{Y}_{\mu} \hat{Y}_{\nu}
$$

with

$$
\rho:=V_{\alpha} V^{\alpha} \rho_{1}, \quad p_{\perp}:=-Y_{\alpha} Y^{\alpha} \rho_{2} .
$$

The transformation in Eq.(23) constrains the new density and transverse pressure to obey

$$
\rho_{1}+\rho_{2}=\rho-p_{\perp}
$$

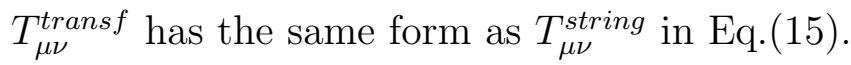

If the two fluids have the same velocity, $\hat{u}_{\mu}=\hat{w}_{\mu}$, then the two-fluid stress-energy becomes a single fluid with a radial stress.

$$
T_{\mu \nu}^{2 \text { fluid }} \rightarrow T_{\mu \nu}^{1 \text { fluid }}=\left(\rho_{1}+\rho_{2}\right) \hat{u}_{\mu} \hat{u}_{\nu}+p_{r} \hat{r}_{\mu} \hat{r}_{\nu}
$$

The density of this string fluid is just the sum of the two densities. In the case $\hat{u}_{\mu}=\hat{w}_{\mu}$ the transverse pressure is zero. The transverse pressure reflects the different velocities of the fluids in the two-fluid model. When the transverse pressure is zero then $m=c_{1} r+c_{2}$.

Using a multi-fluid model, one can also describe the radial stress as reflecting a velocity difference between two dusts so that the complete stress-energy tensor (15) could be described by a three-fluid dust model with all of the dusts moving with different velocities. Which of the interpretations discussed in this section is most likely depends on the actual physical situation being modeled. 


\section{DIFFUSIVE TRANSPORT}

Diffusion has been a seminal process for the development of our understanding of many modern systems. Since the work of Einstein [16], Smoluchowski [17], and Langevin [18], the ideas implicit in diffusion have found new application in areas as diverse as the behavior

of stock option values to cosmic strings. Vilenkin [19] has introduced diffusion into the description of cosmic strings by characterizing string evolution as the formation of Brownian trajectories. This has also been discussed by Bennett [20]. Diffusion is playing an important part in the growth of our understanding of fluctuations in quantum gravity [21], 22] and in the very early life of our universe. For example, Watabiki [23] has used the classical diffusion equation to characterize diffusion times in fractal quantum gravity [24], [25]. Diffusion may also play a role in eternal inflation models where inflation field fluctuations can be modeled as random walks [26]. The path integral technique, developed by Norbert Weiner [27] to describe diffusive processes, has become an essential part of the modern view of quantum mechanics.

String collisions, unlike point particle collisions, do not occur at a single spacetime point (interaction vertex). The two-surface picture of strings requires the collision (interaction) to be a curve on a world-sheet. Observers in different Lorentz frames will see the interaction occurring at different points along the curve. Statistically, the coarse-grained picture of phase space for a set of collisions hides the lack of Lorentz invariance of a single collision. We assume string diffusion is like point particle diffusion in that the number density diffuses from higher numbers to lower according to

$$
\partial_{u} n=\mathcal{D} \nabla^{2} n
$$

where $\nabla^{2}=r^{-2}(\partial / \partial r) r^{2}(\partial / \partial r)$ and $\mathcal{D}$ is the positive coefficient of self-diffusion (which we henceforth take to be constant). Classical transport theory derives the diffusion equation by starting with Fick's law

$$
\vec{J}_{(n)}=-\mathcal{D} \vec{\nabla} n
$$


where $\vec{\nabla}$ is a purely spatial gradient. Then 4-current conservation $J_{(n) ; \mu}^{\mu}=0$, where

$$
\begin{aligned}
J_{(n)}^{\mu} \partial_{\mu} & =\left(n, \vec{J}_{(n)}\right) \\
& =n \partial_{u}-\mathcal{D}(\partial n / \partial r) \partial_{r}
\end{aligned}
$$

yields the diffusion equation (28). We label the 4-current $J_{(n)}$ to indicate $n$ diffusion but we could have also written $J_{(\rho)}$ since the string number density and string fluid density must be related by $\rho=M_{s} n$ where $M_{s}$ is the constant mass of the string species. $M_{s}$ must be a multiple of the Planck mass since it is only over Planck length scales that point particles resolve into strings.

By rewriting the $T_{\mu \nu}$ components (16a) and (16b) as $\dot{m}=-4 \pi r^{2} \psi$ and $m^{\prime}=4 \pi r^{2} \rho$, we can write the integrability condition for $m$ as

$$
\dot{\rho}+r^{-2} \partial_{r}\left(r^{2} \psi\right)=0 .
$$

If we compare the diffusion equation (28) $(n$ replaced by $\rho$ )

$$
\dot{\rho}=\mathcal{D} r^{-2} \partial_{r}\left(r^{2} \partial \rho / \partial r\right)
$$

with $\dot{\rho}$ in Eq.(31) we obtain

$$
\dot{m}=4 \pi \mathcal{D} r^{2} \partial \rho / \partial r
$$

Thus solving the diffusion equation for $\rho$ and then integrating those solutions to obtain $m$ provides exact Einstein solutions which can be interpreted as either anisotropic fluids or diffusing string fluids.

There are some analytic solutions of Eq.(32):

$$
\begin{aligned}
& \rho=\rho_{0}+k_{1} / r, \\
& \rho=\rho_{0}+\left(k_{2} / 6\right) r^{2}+k_{2} \mathcal{D} u, \\
& \rho=\rho_{0}+k_{3}(\mathcal{D} u)^{-3 / 2} \exp \left[-r^{2} /(4 \mathcal{D} u)\right], \\
& \rho=\rho_{0}+\left(k_{6} / r\right) \exp \left(-k_{4}^{2} \mathcal{D} u\right)\left[\sin \left(k_{4} r\right)+k_{5} \cos \left(k_{4} r\right)\right] .
\end{aligned}
$$


Solutions (34) and (36) appear in [7]. In addition to the solutions above, we have obtained a number of analytic solutions using well known similarity techniques. Those solutions will be presented elsewhere. The physical behavior of the density solutions provides a variety of atmospheric models.

Solution (34) describes an atmosphere with a simple drop off in radius and no time dependence. $k_{1}$ must be positive to avoid negative densities. $\rho_{0}$ is the density at spatial infinity.

The density described by equation (35) has two very different behaviors. For $k_{2}>0$, the density is not physically realistic. It increases with radius and grows with time. However, for $k_{2}<0$, the density decreases with radius and has a zero indicating a bounded string atmosphere. Since we are working with a string fluid whose equation of state is $\rho=-p_{r}$, the boundary also has zero radial pressure. The position of the boundary moves inward as $\mathcal{D} u$ increases; the extent of the atmospheric shell decreases with time.

The third density solution, Eq.(36), requires $k_{3}>0$ for positive densities. The solution begins with a high central density which falls off with radius. As time progresses the density decreases to the constant value $\rho_{0}$, the density at spatial infinity.

The fourth density solution, Eq.(37), models a complete array of atmospheric behaviors. For example, the parameter choices $k_{6} / \rho_{0}=10, k_{4}=k_{5}=1$, describe an atmosphere that starts with a string boundary at $\mathcal{D} u=1$ and as time progresses becomes an unbounded string cloud. Other parameter choices model atmospheric shells which are always unbounded. Parameters $k_{5}$ and $k_{6}$ must be positive for positive densities.

\section{DIFFUSIVE MASS SOLUTIONS}

\section{A. Analytic solutions}

Upon integrating $m^{\prime}=4 \pi r^{2} \rho$ and $\dot{m}=4 \pi \mathcal{D} r^{2} \partial \rho / \partial r$ we obtain , in the same order as the densities above, the following 


$$
\begin{aligned}
& m(u, r)=m_{0}+(4 \pi / 3) r^{3} \rho_{0}+2 \pi k_{1}\left(r^{2}-2 \mathcal{D} u\right) \\
& m(u, r)=m_{0}+(4 \pi / 3) r^{3} \rho_{0}+(4 \pi / 3) r^{3} k_{2}\left(\mathcal{D} u+r^{2} / 10\right), \\
& m(u, r)=m_{0}+(4 \pi / 3) r^{3} \rho_{0}+16 \pi k_{3}\left[-\eta \exp \left(-\eta^{2}\right)+(\sqrt{\pi} / 2) \operatorname{erf}(\eta)\right] \\
& m(u, r)=m_{0}+(4 \pi / 3) r^{3} \rho_{0}+\left(4 \pi k_{6} / k_{4}^{2}\right) \exp \left(-k_{4}^{2} \mathcal{D} u\right) B
\end{aligned}
$$

where $\eta:=r(4 \mathcal{D} u)^{-1 / 2}$, and $B=\sin \left(k_{4} r\right)-k_{4} r \cos \left(k_{4} r\right)+k_{5}\left[\cos \left(k_{4} r\right)+k_{4} r \sin \left(k_{4} r\right)\right]$. Solutions (38) and (40) appear in [7].

If parameters $k_{1}, k_{2}, k_{3}$, and $k_{6}$ are zero then all the mass solutions above become the static solution $m_{0}+(4 \pi / 3) r^{3} \rho_{0}$. This is the mass for the isotropic string fluid described by Eq.(19).

\section{B. Interpreting the mass}

Diffusive transport has a conserved four-current for the density

$$
J^{\mu} \partial_{\mu}=\rho \partial_{u}-\mathcal{D}(\partial \rho / \partial r) \partial_{r}
$$

which can be written in terms of the null tetrad

$$
J^{\mu}=\rho n^{\mu}+[(A / 2) \rho-\mathcal{D}(\partial \rho / \partial r)] l^{\mu}
$$

When $J_{; \mu}^{\mu}=0$ is integrated over a four-volume, Stokes theorem casts the integral onto the bounding three-surfaces:

$$
\int_{R_{4}} J_{, \mu}^{\mu} \sqrt{-g} d^{4} x=\oint_{\partial R_{4}} J^{\mu} \sqrt{-g} d S_{\mu}
$$

where we integrate over $u=$ const null three-surfaces $\mathcal{N}_{2}$ and $\mathcal{N}_{1}$ with $d S_{\mu}=l_{\mu} d r d \vartheta d \varphi$, and $r=$ const timelike three-surfaces $\Sigma_{2}$ and $\Sigma_{1}$ with $d S_{\mu}=\left(n_{\mu}-\frac{A}{2} l_{\mu}\right) d u d \vartheta d \varphi$. The four-volume $R_{4}$ can be pictured as a tube surrounding a timelike cylinder containing the central source. An orthogonal cross-section of $R_{4}$ would have sides $\mathcal{N}_{1}, \mathcal{N}_{2}, \Sigma_{1}, \Sigma_{2}$, forming a rhomboid with $\Sigma_{1}$ bounding $R_{4}$ away from the central source. Thus 


$$
\int_{\mathcal{N}_{2}-\mathcal{N}_{1}} J^{\mu} l_{\mu} \sqrt{-g} d r d \vartheta d \varphi+\int_{\Sigma_{2}-\Sigma_{1}} J^{\mu}\left(n_{\mu}-\frac{A}{2} l_{\mu}\right) \sqrt{-g} d u d \vartheta d \varphi=0
$$

with specific form

$$
\int_{\mathcal{N}_{2}-\mathcal{N}_{1}} \rho \sqrt{-g} d r d \vartheta d \varphi-\int_{\Sigma_{2}-\Sigma_{1}} \mathcal{D}(\partial \rho / \partial r) \sqrt{-g} d u d \vartheta d \varphi=0 .
$$

To first understand the string mass, we examine a static string fluid. Metric (11) includes static string fluids when $m(u, r)$ is restricted to $m(r)$. We can use the density solution Eq.(34) with $\mathcal{D}=0$ so that there is no diffusion. For $\rho=\rho_{0}+k_{1} / r$, we have

$$
4 \pi \int_{\mathcal{N}_{2}-\mathcal{N}_{1}}\left(\rho_{0} r^{2}+k_{1} r\right) d r=0 .
$$

Thus the string mass is static with $m_{\text {string }}(r)=\int_{\mathcal{N}_{1}}=\int_{\mathcal{N}_{2}}$ :

$$
m_{\text {string }}(r)=m_{0}+4 \pi\left(\frac{1}{3} \rho_{0} r^{3}+\frac{1}{2} k_{1} r^{2}\right)
$$

on null surface $\mathcal{N}_{1}$ and at a later time on $\mathcal{N}_{2}$. This is the mass in Eq.(38) with $\mathcal{D}=0$.

Now consider the case with time dependence where the mass is $m(u, r)$ and there is a Vaidya fluid of short photons. Again using $\rho=\rho_{0}+k_{1} / r$, we find

$$
4 \pi \int_{\mathcal{N}_{2}-\mathcal{N}_{1}}\left(\rho_{0} r^{2}+k_{1} r\right) d r+4 \pi \int_{\Sigma_{2}-\Sigma_{1}} k_{1} \mathcal{D} d u=0
$$

This yields the total mass of strings and null fluid in Eq.(38) where

$$
m(u, r)=\int_{\mathcal{N}_{2}}-\int_{\Sigma_{1}}=\int_{\mathcal{N}_{1}}-\int_{\Sigma_{2}}
$$

We use our knowledge of the static case to identify the string mass as that part of the total mass integrated over the null three-surface $\mathcal{N}$, and identify the flux through the timelike surface $\Sigma$ as resulting from both the energy carried by the Vaidya photons and the diffusing strings. Photons enter $R_{4}$ from the central source through $\Sigma_{1}$ while string bits diffuse through $\Sigma_{1}$ toward the source, with the opposite happening at $\Sigma_{2}$. There the photons leave $R_{4}$ while string bits enter. Thus $m(u, r)=m_{\text {string }}+m_{\text {flux }}$ where

$$
\begin{aligned}
m_{\text {string }} & =4 \pi\left(\frac{1}{3} \rho_{0} r^{3}+\frac{1}{2} k_{1} r^{2}\right) \\
m_{\text {flux }} & =-4 \pi k_{1} \mathcal{D} u
\end{aligned}
$$

Of course $m_{\text {string }}$ need not be static. The density examples with $\rho(u, r)$ will have $m_{\text {string }}(u, r)$. 


\section{HORIZONS}

The topological two-spheres $(\vartheta, \varphi)$ nested in an $r=$ const surface at time $u$ have outgoing null geodesic normal $l^{\mu}$ and incoming null geodesic normal $n^{\mu}$. The spheres become trapped surfaces when both $l^{\mu} r_{, \mu}$ and $n^{\mu} r_{, \mu}$ are positive (negative for +2 signature). The marginally trapped surface is the outer boundary of all trapped surfaces at time $u$, and the apparent horizon is the time history of the marginally trapped surface.

Here $l^{\mu} r_{, \mu}=1$. The fluid atmosphere has $n^{\mu} r_{, \mu}=-A / 2$, negative until $m(u, r) \geq r / 2$. At that time the mass has become compact enough to trap light.

We will analyze mass expression (38) for trapped surfaces. With $L_{0}:=2 m_{0}, L_{1}^{-3}:=$ $\left(4 \pi \rho_{0}\right) /\left(3 m_{0}\right), L_{2}^{-2}:=2 \pi k_{1} / m_{0}$, and $1 / T_{0}:=4 \pi k_{1} \mathcal{D} / m_{0}$, we search for zeros of the polynomial

$$
\frac{r^{3}}{L_{1}^{3}}+\frac{r^{2}}{L_{2}^{2}}-\frac{r}{L_{0}}+1-\frac{u}{T_{0}}=0
$$

at time $u=$ const. The four parameters $\left(T_{0}, L_{0}, L_{1}, L_{2}\right)$ are positive. Suppose there is a root at $r=\alpha L_{0}, \alpha>0$. Then, at $u=0$, Eq.(49) becomes

$$
\alpha^{3}\left(L_{0} / L_{1}\right)^{3}+\alpha^{2}\left(L_{0} / L_{2}\right)^{2}+1-\alpha=0
$$

or

$$
\alpha^{2}\left(2 m_{0}\right)^{2}\left[\alpha\left(8 \pi \rho_{0} / 3\right)+2 \pi k_{1} / m_{0}\right]+1-\alpha=0
$$

If $k_{1}$ and $\rho_{0}$ are zero then $\alpha=1$ and we find the Schwarzschild horizon at $r=2 m_{0}$. For

non-zero parameters Eq.(50) has no real roots, which is consistent with the outgoing shortwavelength photons.

At time $u=0$ and for some short time after $1-u / T_{0}$ is positive. After more time $\Delta u$, $1-u / T_{0}$ becomes negative and then there is only one sign change in Eq.(49). Descartes' rule of signs tells us that, for $u>\Delta u$, there is at most one real root of Eq.(49) and the fluid will have a trapped surface at $r=2 m(u, r)$. 


\section{CONCLUSION}

That $\hat{v}^{\mu}$ is hypersurface orthogonal implies $\hat{v}_{\mu} d x^{\mu}=f(u, r) d t$. If metric (目) were the Schwarzschild metric then $A=1-2 m_{0} / r, u=t-r-2 m_{0} \ln \left(r-2 m_{0}\right)$, and $\hat{v}_{\mu} d x^{\mu}=A^{-1 / 2} d t$. Next in simplicity is the Vaidya metric, where $A$ of metric (1) would be $A=1-2 m(u) / r$. The Vaidya metric is not static and one cannot coordinate transform back to Schwarzschild. With the Vaidya metric $r-2 m(u)$ is a spacelike hypersurface lying outside the local null cone [29], unlike the Schwarzschild hypersurface $r-2 m_{0}$ which is null.

The traditional view of the Vaidya metric places it outside a spherical star which is losing mass via Vaidya's short-wavelength photons. Vacuum Schwarzschild geometry is joined smoothly to Vaidya at its radiative boundary.

The system described here with $m(u, r)$ continues to have $\hat{v}^{\mu}$ hypersurface orthogonal, so the $t$ in $\hat{v}_{\mu} d x^{\mu}=f(u, r) d t$ labels spacelike hypersurfaces with energy-momentum given by Eq.(15) above. Because of the implicit equation of state, $\rho+p_{r}=0$, we interpreted the fluid as an "atmosphere" of open strings which form a string fluid. This view has also been discussed by 't Hooft [6] and Maldacena [5]. This fluid was taken as a source of energymomentum. As a modeling choice, we took the strings to interact diffusively with energy carried away by short-wavelength photons. It was necessary to use the approximation of short-wavelength photons since $\left(2 \dot{m} / r^{2}\right) l_{\mu} l_{\nu}$ is not an exact solution of Maxwell's equations.

Quantum field theory requires the quantum vacuum to be Lorentz invariant 30, which constrains the energy-momentum tensor on a Minkowski background to have the form $T_{\mu \nu}=$ $\rho \eta_{\mu \nu}$, and the energy-density to transform as

$$
\rho^{\prime}=\frac{\rho+p\left(v^{2} / c^{2}\right)}{1-\left(v^{2} / c^{2}\right)}
$$

under change of inertial frame. The string equation of state $p_{r}=-\rho$ satisfies the required transformation property and so the vacuum outside a relativistic star could indeed include a string atmosphere.

This work necessarily forms an incomplete picture of the evolution of the astrophysical system we model here. Strings may exist at the Planck length scale, with a large number 
of them possibly providing a macroscopic classical string, and forming a visible atmosphere until they lie within a trapped surface.

\section{ACKNOWLEDGMENTS}

E.N. Glass was partially supported by an NSERC of Canada grant. Computations were verified using MapleV.4 (Waterloo Maple Software, Waterloo, Ontario) and GRTensorII rel 1.59 (P. Musgrave, D.Pollney, and K. Lake, Queens University, Kingston, Ontario).

[1] Mitchell D and Turok N 1987 Nuc. Phys. B294 1138

[2] Sen A 1998 "Developments in Superstring Theory", hep-th/9810356

[3] Gott J R 1985 Ap. J. 288422

[4] Larsen F 1997 Phys. Rev. D56 1005

[5] Maldacena J M 1996 Black Holes in String Theory, Princeton University Dissertation

[6] 't Hooft G 1997 "The Self-Screening Hawking Atmosphere", presented at Strings97, Amsterdam gr-qc/9706058

1997 "Distinguishing causal time from Minkowski time and a model for the black hole eigenstates" gr-qc/9711053

[7] Glass E N and Krisch J P 1998 Phys. Rev. D57 R5945

[8] Vaidya P C 1953 Nature 171260

[9] Letelier P S 1979 Phys. Rev. D20 1294

[10] Letelier P S 1981 Nuov. Cim. 63B 519

[11] Hiscock W A 1977 Phys. Rev. D15 3054 
[12] Garfinkle D, Horowitz G and Strominger A 1991 Phys. Rev. D43 3140

[13] Parthasarathy R and Viswananthan K S 1992 Phys. Lett. B400 27

[14] Kar S 1997 Phys. Rev. D55 4872

[15] Letelier P S 1980 Phys. Rev. D22 807

[16] Einstein A 1905 Ann. Physik 17549

[17] Smoluchowski M v 1907 Ann. Physik 21756

[18] Langevin P 1908 Comptes Rendus 146530

[19] Vilenkin A 1981 Phys. Rev. D24 2082

[20] Bennett D P 1986 Phys. Rev. D33 872

[21] Percival I C 1995 Proc. Roy. Soc. A451 503

[22] Percival I C and Strunz W T 1997 Proc. Roy. Soc. A453 431

[23] Watabiki F Y 1996 Frontiers in Quantum Field Theory (World Scientific, Singapore p. 158)

[24] Ambjorn J and Watabiki Y 1995 Nuc. Phys. B 445129

[25] Ambjorn J, Jurkiewicz J and Watabiki Y 1995 Nuc. Phys B 454313

[26] Borde A and Vilenkin A 1997 Phys. Rev. D56 717

[27] Greiner W and Reinhardt J 1996 Field Quantization (Springer-Verlag, Berlin p. 342)

[28] Misner C W and Sharp D H 1964 Phys. Rev. 136 B571

[29] Lindquist R W, Schwartz R A and Misner C W 1965 Phys. Rev. 137 B1364

[30] Lima J A S and Maia Jr. A 1995 Phys. Rev. D52 5628 